\title{
Sommerschule als Weiterentwicklungschance für Schüler*innen und Student*innen?
}

\section{Ein kurzer Einblick}

DOI: https://doi.org/10.53349/sv.2021.i1.a61

\begin{abstract}
Im folgenden Artikel teile ich meine Erfahrungen als Studentin des BA Lehramts der Germanistik und der katholischen Theologie in der Sommerschule 2020. Mein Schulstandort befand sich im Bundesgymnasium Zehnergasse Wiener Neustadt und unter der Leitung von Dir. HR Mag. Dr. Werner Schwarz durfte ich meine Fähigkeiten als Lehrende unter Beweis stellen. Eine praxisnahe Chance, wie es die Sommerschule meiner Meinung nach ist, sollte man als Studierende*r des Lehramts im weitesten Maße ausnutzen. Denn sehr selten bekommt man die Chance, sich zwei Wochen lang intensiv mit einund derselben Klasse oder auch Schülergruppe auseinanderzusetzen.
\end{abstract}

\section{Aller Anfang ist schwer}

Die tatkräftige Unterstützung durch die Lehrer*innen und den Direktor, während der Vorbereitung auf das zweiwöchige Projekt, ließen mich mit einem durchaus positiven Gefühl in die Sommerschule starten. Meine Klasse bestand aus 14 Schüler*innen im Alter von 13 bis 15 Jahren. Das Konzept „Märchen” als tonangebendes Thema musste ich jedoch durch diesen Umstand schon am ersten Tag aufgeben und mit dem Stoffgebiet des Heldentums ersetzen. Die ersten zwei Tage waren sowohl für mich als auch für die Lernenden eine Geduldsprobe und Herausforderung, weil beide Seiten die Dynamiken und Vorgangsweisen verinnerlichen und austesten mussten.

Die Diversität der Schüler*innengruppe forderte überdies auch diverse Methoden und Herangehensweisen. Der Fokus lag auf der grundlegenden Grammatik der deutschen Sprache 
und einer fundierten, angemessenen Diskussions- und Gesprächskultur, welche von den Schüler*innen selbst etabliert und aufgeschrieben wurde.

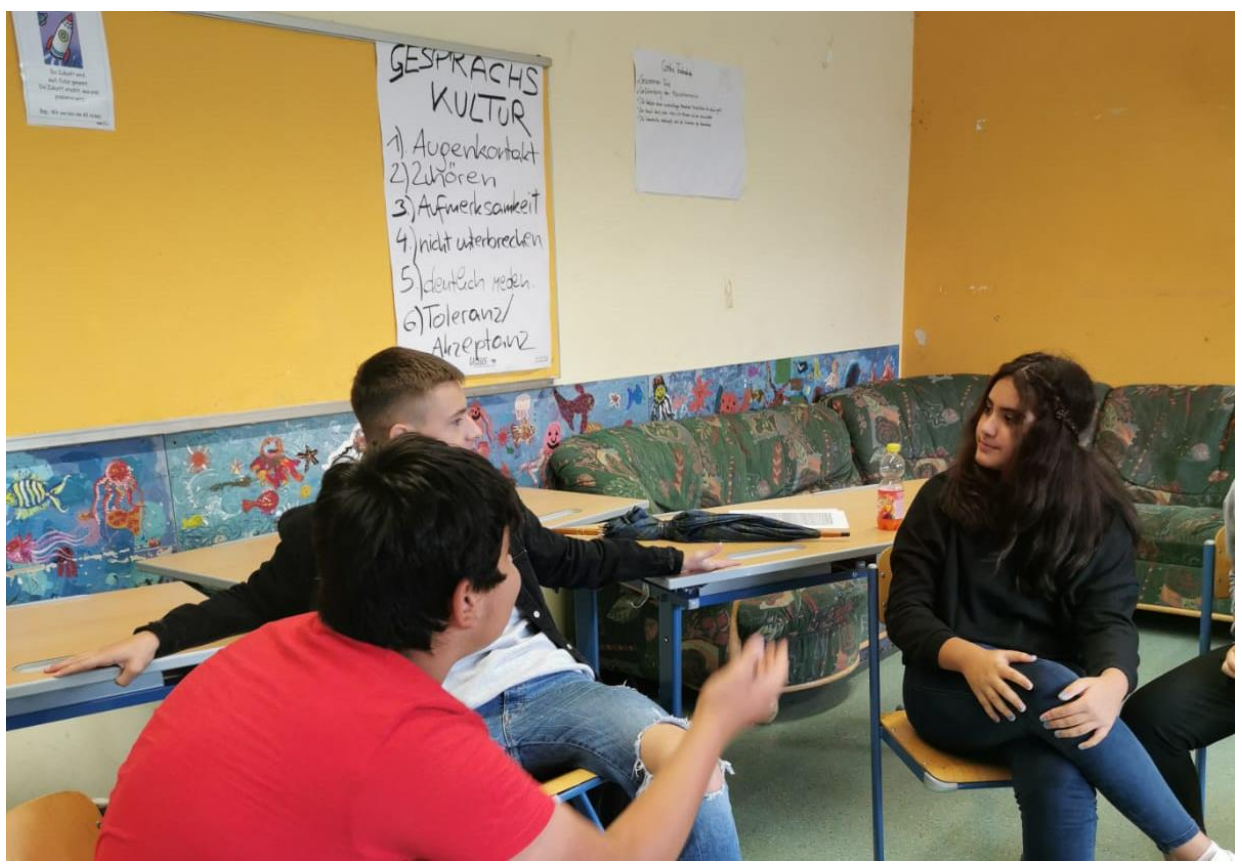

Abbildung 1: Gesprächskultur (Foto: privat)

\section{Zwei Wochen voller Chancen}

Nach den ersten zwei Tagen wurden sowohl die Gruppendynamik als auch das Klassenklima erheblich entspannter und offener. Zu meiner Überraschung kamen die Lernenden von sich aus mit ihren Problemen und Schwächen auf mich zu und gemeinsam konnte ein detaillierter Plan für die kommenden Tage formuliert werden. Besonderen Anklang fanden die Plenumsund Podiumsdiskussionen im Kontext „Helden im Rechtssystem“ und „Helden auf der Leinwand“. Der anfängliche Jammer seitens der Schüler*innen bezüglich der Grammatikaufgabenstellungen konnte durch rasche Erfolgserlebnisse und gegenseitiges Feedback ausgelotet werden. Ein Lerntagebuch gab mir schlussendlich Auskunft über die Sicht aller Jugendlichen bezugnehmend ihrer Fortschritte und damit auch Ideen für mögliche weitere Übungen.

Der Start der Woche stellte sich für mich als besonders prägend dar, weil ich mich erstmalig alleine um eine Klassengemeinschaft kümmern musste. Meine Ziele und Ansprüche an mich selbst trieben mich dazu, sehr viel mehr vorzubereiten als schlussendlich erarbeitet werden konnte. Als Lehrperson konnte ich durch diese neuen Herausforderungen wachsen und einen adäquaten Einblick in die Abläufe des Lehrer*innen-Daseins gewinnen.

Obwohl fast alle Jugendlichen von ihren Erziehungsberechtigten zur Sommerschule gedrängt worden waren, konnte schon nach kurzer Zeit ein Motivationsschub ausgelöst werden, der 
fast die gesamte Gruppe mit sich zog. Neue Freundschaften zwischen Schüler*innen aus verschiedenen Schulen wurden geschlossen und gepflegt, unabhängig von Ethnie oder Alter.

\section{Resümee}

Die Sommerschule war nicht nur für die Schüler*innen ein Lernerlebnis, sondern vor allem auch für mich als Lehrperson. Aufgrund weniger Praxisstunden während des Studiums muss man sich erst als Lehrende* $r$ finden und einen Weg einschlagen, der einem selbst, aber auch den Vorstellungen der Jugendlichen entgegenkommt. Ich denke, dass die Lernenden sehr wohl eine attraktive Auffrischung und Erweiterung ihrer Kenntnisse erfahren durften, großreichende Probleme und Schwerpunkte können meines Erachtens aber nicht in so kurzer Zeit ausgemerzt werden.

Die universitäre Vorbereitung fand in Form von Tageskursen statt, in denen man sowohl Literatur als auch praxisnahe Beispiele auf- und vorbereitete. Das Problem bestand jedoch darin, dass der Kontaktaufbau zu den Schulen erst nach diesem Kurs geschah und man somit nicht viel über die Schüler*innen, welche man schlussendlich betreut hat, wusste. Dies war der Grund, warum ich meine erste Planung komplett überdenken und neugestalten musste. Obwohl uns seitens der Regierung gesagt wurde, dass Student*innen immer im Tandem unterrichten werden, um nicht überfordert zu sein, war das bei mir und sämtlichen Kommiliton*innen nicht der Fall. Die große Mehrheit musste sich alleine einer Klasse widmen und wurde im besten Szenario von den Lehrer*innen vor Ort unterstützt, wie es bei mir der Fall war.

Das zweiwöchige Erlebnis hat mich im Nachhinein sehr positiv in meiner Entscheidung, eine Lehrkraft zu werden, bestärkt und ich möchte diese Erfahrung allen Studierenden nahelegen, die sich erstens in der Praxis so richtig beweisen wollen und zweitens einen groben Einblick in das Leben einer Lehrperson erhalten möchten. Insgesamt kann man die Sommerschule als gelungenes Projekt ansehen, dessen jährlicher Wiederholung ich nur lautstark zustimmen kann.

\section{Autorin}

\section{Viola Frieda Susanne Mayer}

studiert die Fächer Germanistik und katholische Theologie an der Hauptuniversität Wien im achten Semester. Die Germanistik gehört zu ihren großen Leidenschaften, wobei sie großen Wert auf die künstlerische Auslebung des Wortes legt. Zukünftig möchte sie Schüler*innen inspirieren und ihnen zu großen Erfolgen verhelfen. Kontakt: violamayer98@gmail.com 